\title{
IMPLANTAÇÃO DA AUDITORIA OPERATIVA EM UMA AUTARQUIA MUNICIPAL
}

Vanessa Moraes Liberatti ${ }^{1}$

Danielli Rafaeli Candido Pedro ${ }^{2}$

Raquel Grozd ${ }^{2}$

Paloma de Souza Cavalcante Pissinati

Isabel Cristina Kowal Olm Cunha ${ }^{3}$

Maria do Carmo Fernandez Lourenço Haddad² https://orcid.org/0000-0001-9883-6444

https://orcid.org/0000-0003-4141-1220

https://orcid.org/0000-0002-5816-8215

https://orcid.org/0000-0001-9050-4330

https://orcid.org/0000-0001-6374-5665

https://orcid.org/0000-0001-7564-8563

Objetivo: Relatar a experiência do processo de implantação da auditoria operativa na Autarquia Municipal de Saúde de um município de grande porte do Sul do Brasil. Método: Trata-se de um relato de experiência, realizado por meio da análise de documentos internos e públicos da referida Autarquia de Saúde, elaborados durante os anos de 2004 a 2018. Resultados: Identificou-se um aumento no número de auditores, ampliação da quantidade de serviços credenciados ao Sistema Único de Saúde que passaram a ser auditados, implantação e implementação de relatórios de auditoria, auxilio no desenvolvimento de manuais e protocolos, além de melhoria na qualidade da assistência prestada. Conclusão: A implantação do serviço de auditoria operativa é de suma importância na gestão do SUS, não só nos serviços de média e alta complexidade, mas também na atenção primária e demais setores e serviços de assistência à saúde. Descritores: Sistema Único de Saúde; Enfermagem; Auditoria de Enfermagem.

\section{IMPLEMENTATION OF OPERATIVE AUDIT IN A MUNICIPALAUTHORITY}

Objective: To report the experience of the process of implementation of the operative audit in the Municipal Health Council of a large municipality. Method: This is an experience report, conducted through the analysis of internal and public documents of the Municipal Health Authority of a large municipality in southern Brazil, prepared during the years 2004 to 2018. Results: Was identified an increase in the number of auditors, expansion of the number of accredited services to the Unified Health System that are being audited, implementation and implementation of audit reports, assistance in the development of manuals and protocols, as well as improvement in the quality of care provided. Conclusion: The implementation of the operative audit service is of paramount importance in Unified Health System management, not only in medium and high complexity services, but also in primary care and other health care sectors and services. Descriptors: Unified Health System; Nursing; Nursing Audit.

\section{IMPLEMENTACIÓN DE LAAUDITORÍA OPERATIVA EN UNAAUTORIDAD MUNICIPAL}

Objetivo: Informar la experiencia del proceso de implementación de la auditoría operativa en el Consejo Municipal de Salud de un municipio grande. Método: Este es un informe de experiencia, realizado mediante el análisis de documentos internos y públicos de la Autoridad Municipal de Salud de un municipio grande en el sur de Brasil, preparado durante los años 2004 a 2018. Resultados: Identificamos un aumento en el número de auditores, la expansión del número de servicios acreditados para el Sistema Único de Salud que están siendo auditados, la implementación y la implementación de informes de auditoría, asistencia en el desarrollo de manuales y protocolos, así como la mejora en la calidad de la atención brindada. Conclusión: La implementación del servicio de auditoría operativa es de suma importancia en la gestión del Sistema único de Salud, no solo en los servicios de complejidad media y alta, sino también en la atención primaria y otros sectores y servicios de atención médica.

Descriptores: Sistema único de Salud; Enfermería; Auditoría de enfermería.

${ }^{1}$ Universidade Estadual de Maringá, PR.

2 Universidade Estadual de Londrina,PR.

${ }^{3}$ Universidade Federal de São Paulo,SP.

Autor Correspondente: Vanessa Moraes Liberatti - Email: vanessa_liberatti@hotmail.com 


\section{INTRODUÇÃO}

A saúde é considerada um dos setores de atividade econômica, dominante em vários países. Nos últimos 40 anos observou-se um rápido aumento nos gastos relacionados à saúde, o que exige dos gestores conhecimentos de suas necessidades e especificidades de forma a garantir uma gestão qualificada(1) , e otimização dos recursos financeiros para que opere com eficácia e eficiência. ${ }^{(2)}$

Além da preocupação financeira, onde se procura trabalhar com redução de custos atrelada à excelência de resultados, existe a necessidade de priorizar a satisfação dos usuários e a qualidade da assistência. (3) Nesse contexto, a auditoria configura-se como uma ferramenta de gestão na execução desse processo, estratégia essencial no gerenciamento dos serviços de saúde. ${ }^{(4)}$

Existem diferentes classificações da auditoria em saúde, sendo finalidade, previsibilidade, causa e tipo. Quanto à finalidade, ela pode ser de regularidade, operacional ou de desempenho e integrada. A previsibilidade caracteriza as auditorias em programadas e não programadas. Quanto à causa, a auditoria pode ser regular ou ordinária e especial ou extraordinária. Relacionado ao tipo, ela pode ser analítica, operativa, de gestão e contábil. A execução envolve auditoria prospectiva ou prévia, retrospectiva e concorrente. Quanto à forma, ela pode ser interna ou de primeira parte, externa ou de segunda parte. ${ }^{(5)}$

No Brasil, o Sistema Único de Saúde (SUS) tem desenvolvido estratégias de racionalização do uso dos recursos, expressas no sistema de regulação(1) e implantado serviços inovadores de auditoria, tais como o serviço de auditoria operativa em alguns setores específicos.

A operacionalização da auditoria nos serviços de saúde, em especial, da auditoria operativa, proporciona que o auditor esteja em contato frequente com os prestadores, com a equipe e com os pacientes. Esta estratégia facilita o cumprimento das metas contratualizadas, esclarece dúvidas pontuais e permite o acompanhamento in loco a assistência prestada. ${ }^{(6)}$

No âmbito da gestão em saúde, o enfermeiro se destaca na execução da auditoria, pois sua formação permite apreender conteúdos direcionados à gerência de serviços. ${ }^{(7)}$ Além disso, em seu processo de trabalho atua diretamente na liderança, tendo a auditoria como atividade inerente para o monitoramento das ações e indicadores pactuados nos diferentes níveis de complexidade em que atue.

Diante disso, o objetivo deste estudo foi relatar a experiência do processo de implantação da auditoria operativa de Enfermagem na Autarquia Municipal de Saúde de um municipio de grande porte.

\section{METODOLOGIA}

\section{Tipo de estudo}

Trata-se de um relato de experiência, do processo de implantação de uma auditoria operativa em uma Autarquia Municipal de Saúde.

\section{Local do estudo}

Em uma Autarquia Municipal de Saúde de um município de grande porte, com gestão plena, localizado no Sul do Brasil.

\section{Coleta de dados}

Realizou-se a análise de documentos internos e públicos, que incluíram diários oficiais, ofícios, contratos, editais, atas de reuniões e relatórios de auditoria dos anos de 2004 a 2018.

Foram analisados 105 documentos internos. A coleta de dados foi realizada no período de novembro de 2018 a janeiro de 2019 buscou-se nos documentos informações como registros em atas e ofícios da implantação do serviço de auditoria, relatórios para analisar a trajetória evolutiva do serviço, tanto quantitativamente, quanto qualitativamente, contratos com as instituições onde existia menção ao serviço de auditoria, editais e diários oficiais do município que dispunham sobre os requisitos para o cargo de enfermeiro auditor, bem como o número de contratações, podendo então descrever o processo de implantação da auditoria e relatar a experiência com esse processo.

\section{Procedimentos éticos}

Obedecendo as questões éticas, obteve-se o consentimento da organização para esta descrição e aprovação do projeto por Comitê de Ética em Pesquisa, parecer $\mathrm{n}$ 의.702.126/2016.

\section{RESULTADOS}

O município em estudo está situado no Sul do Brasil e em 2018 possuía uma população estimada de 563.943 habitantes. ${ }^{(8)}$ Sempre foi referência na área da saúde a nivel nacional, tendo destaque na saúde pública desde a implantação das Unidades Básicas de Saúde (UBS) da Estratégia Saúde da Família (ESF). Atualmente, é referência para aproximadamente um milhão de habitantes, que compõem os 21 municípios da 17ạa Regional de Saúde. ${ }^{(9)}$

A Autarquia Municipal de Saúde possui contratualização 
com diversos serviços de saúde, tanto públicos, filantrópicos e privados. Existe contratualização com três hospitais terciários, dois secundários, um Centro de Assistência de Alta Complexidade em Oncologia (CACON), um hospital especializado em saúde mental, um hospital especializado em oftalmologia e um Consórcio Intermunicipal de Saúde, além de sete clínicas de reabilitação, quatro laboratórios, cinco clínicas de imagem, dentre outros. Foi pioneira na implantação da auditoria em saúde, conforme a legislação vigente na época (2014) e se destacou por já implementar e criar cargos específicos, como a equipe de auditoria operativa. ${ }^{(10)}$

Considerando que a auditoria no SUS é complexa, sendo necessária uma grande quantidade de dados que devem ser extraídos, analisados e interpretados cuidadosamente, pois interesses e responsabilidades diversos ficam em evidência quando se audita a saúde. Destaca-se a necessidade de ter uma equipe de auditores responsável por coletar dados, acompanhar a execução de contratos, analisar as produções e os dados e propor melhorias e modificações sempre que necessário. ${ }^{(4)}$

No que se refere à estrutura organizacional da Autarquia Municipal de Saúde do referido município, inicialmente Diretoria de Auditoria, Controle e Avaliação (DACA), era responsável por auditar e avaliar os hospitais credenciados ao SUS no município. ${ }^{(10)}$

Em meados de 2004, o município enfrentava a superlotação dos hospitais terciários, situação que culminou na determinação de técnicos administrativos para acompanhar o gerenciamento da ocupação dos leitos SUS, por meio dos censos hospitalares. Tempos depois (sem data definida nos documentos), essa função foi designada a uma enfermeira, que visitava os prontos socorros dos hospitais para observar e analisar a ocupação, com o objetivo de avaliar a qualidade da assistência prestada, dando início a execução da auditoria operativa de enfermagem.

Em meados de 2007 a DACA contratou, de forma terceirizada, uma equipe de oito enfermeiros, que visitavam todos os dias os hospitais, acompanhando e registrando, em planilhas, as taxas de ocupação, não apenas de prontos socorros, mas de setores críticos como Unidade de Terapia Intensiva (UTI) adulto, UTI neonatal e UTI pediátrica. A equipe de auditoria passou a utilizar os contratos, assinados previamente entre os gestores municipais e as instituições prestadoras de serviços ao SUS, como instrumento para realizar as visitas de auditoria. O olhar para a qualidade da assistência e metas qualitativas foi incorporado à rotina dos enfermeiros auditores.
Em 2012 a DACA passou a ser denominada Diretoria de Regulação da Atenção à Saúde (DRAS) devido a um processo de reestruturação, com base no Decreto Municipal nㅇ 847/2012 e na Lei Municipal no 11.610/2012. (10) Outra alteração foi feita em relação à equipe de enfermeiros auditores, agora em relação à contratação de profissionais, já que o município teve problemas com a empresa terceirizada que realizava esse serviço. Realizou-se um processo seletivo para contratação de profissionais para compor a equipe. Não havia exigência ainda que o enfermeiro auditor possuisse especialização em auditoria, tratou-se de uma forma de regularizar a contratualização desses profissionais e de aumentar as funções do cargo.

A equipe foi ampliada, para dez enfermeiros, e a carga horária a ser cumprida era de 42 horas semanais. desempenhada tanto em hospitais secundários, quanto terciários. Além da taxa de ocupação hospitalar, incorporou-se a análise de novos indicadores, como a quantidade de cirurgias realizadas, de atendimentos de urgência, a qualidade da assistência prestada e as metas qualitativas contratualizadas.

Em 2013, a fim de regularizar o Serviço de Auditoria Operativa de Enfermagem, considerando a importância que este setor passou a ter no acompanhamento dos contratos com os prestadores de saúde e com a melhora nos indicadores assistenciais qualitativos, foi criado o cargo de enfermeiro auditor/regulador, por meio do Jornal Oficial $n$ o 2169/2013. (11) A carga horária de trabalho exigida passou a ser de 30 horas semanais e o número de vagas foi ampliado para 14 enfermeiros.

Para a criação desse cargo foram determinados requisitos específicos como: possuir diploma de graduação em Enfermagem, registro no respectivo conselho da categoria profissional, carteira nacional de habilitação categoria $B$, residência/especialização em regulação ou auditoria e não possuir vínculo com serviços contratados/ conveniados ao SUS. ${ }^{(12)}$

Agregaram-se também diversas funções ao cargo, como: atuar em atividades de planejamento, elaboração, coordenação, acompanhamento, assessoramento, pesquisa e execução de procedimentos e ações de regulação e auditoria em unidades do sistema municipal e regional de saúde no âmbito do SUS. ${ }^{(12)}$

Em 2014 a nova equipe assumiu suas atividades e passou a desempenhar sua função de forma contínua e definitiva. Desde a implementação oficial da equipe de auditoria, verificou-se diversos resultados, conforme apresentado no Quadro 1. 
Quadro 1. Resultados da implementação da equipe de Auditoria Operativa no Município. Londrina-PR, Brasil. 2019

1. Diminuição dos erros no preenchimento dos documentos oficiais, como AIH e Autorização de Procedimentos Ambulatoriais (APAC) por parte dos hospitais.

2. Maior agilidade em relação aos processos de autorização de procedimentos especiais em pacientes internados, que antes chegavam a cinco dias e que hoje não excede 36 horas.

3. Implantação de emissão de relatórios mensais de produtividade para cada serviço contratualizado.

4. Implantação de Procedimentos Operacionais Padrão (POP) para cada processo de trabalho do serviço de auditoria em enfermagem.

5. Desenvolvimento e implantação de manuais quanto ao atendimento de pacientes SUS.

6. Redução na quantidade de reclamações recebidas pela ouvidoria da Autarquia Municipal de Saúde do Município sobre os serviços prestados pelas instituições contratualizadas com o SUS.

7. Melhora na qualidade dos registros nos prontuários, atendendo as exigências legais preconizadas.

8. Implantação de checklist e questionários de itens de avaliação que subsidiam diariamente as auditorias operativas.

9. Aproximação entre a equipe de auditoria e os prestadores de serviço e amplo desenvolvimento dos auditores mais como profissionais educadores e orientadores, do que agentes punitivos.

10. Capacitações da equipe de enfermeiros auditores, inclusive com profissionais cursando mestrado e doutorado.

11. O setor de auditoria operativa de enfermagem passou a ser campo de estágio para residentes, tanto da área de gerência dos serviços de saúde, quanto de saúde pública.

12. Redução do tempo de espera para realizar cirurgias eletivas.

13. Higienização das filas de espera para cirurgias eletivas, com a atualização das solicitações e exclusões, quando necessário.

14. Passagem diária e em tempo real de informações sobre a ocupação de leitos nos hospitais para as centrais de regulação.

15. Auditoria integrada a outros serviços como: vigilância sanitária, controle social (conselho municipal de saúde), auditoria estadual e federal, dentre outros.

16. Diminuição do tempo de espera de atendimentos ambulatoriais de pacientes SUS, em especial a pacientes oncológicos.

17. Implantação de relatório mensal sobre a tempestividade relacionada as cirurgias eletivas oncológicas.
18. Diminuição dos encaminhamentos de pacientes SUS por meio de fluxos irregulares na urgência/emergência.

19. Implantação da contra referência de pacientes hospitalares em caso de regulação equivocada quanto à complexidade dos niveis primário, secundário e terciário de atendimento.

20. Melhora no cumprimento da política de humanização nos hospitais, com: visita ampliada a pacientes SUS, permanência de acompanhantes mesmo em setores críticos, diminuição do tempo de espera para ter acesso a um leito de internação.

\section{DISCUSSÃO}

Em 2014, com a inserção de enfermeiros especialistas em auditoria, houve um desenvolvimento ainda maior da equipe. Houve a ampliação da auditoria para os serviços ambulatoriais dos hospitais terciários, e setor de emergência, bem como hospitais especializados (um em saúde mental, um CACON e um hospital oftalmológico) e dois serviços de terapia renal substitutiva.

Atualmente, todos os setores assistenciais das instituições são auditados mensalmente. A nível hospitalar, diariamente os enfermeiros auditores visitam todos os setores de internação e de realização de procedimentos (desde centro cirúrgico até setores de procedimentos hemodinâmicos e ambulatoriais), o que representa 100\% de cobertura

Verifica-se atualmente a necessidade cada vez maior da implantação e atuação de serviços de auditoria em instituições de saúde, objetivando a análise rigorosa dos processos, atuando como um indicador quantitativo e qualitativo e potencial estratégia para a redução de custos e riscos aos usuários. ${ }^{(13)}$

É rotina fazer entrevistas com pacientes, de forma aleatória e em casos específicos, de forma direcionada, como para pacientes com longos períodos de internação, nas quais busca-se investigar a qualidade da assistência, além de itens relacionados à humanização, fluxo de acesso ao serviço de saúde, dentre outros necessários.

São auditadas todas as fichas de atendimento de pronto socorro dos hospitais, representando uma média mensal de 9.500 fichas e todos os laudos de Autorização de Internações Hospitalares (AIH), sendo em média 2.526 laudos mensais.

Em caso de identificar qualquer descumprimento de item contratualizado, é gerada uma não conformidade e sempre que necessário é enviado ao prestador um ofício solicitando uma resposta ao problema identificado. A não conformidade pode estar relacionada a anotações 
incompletas, recursos humanos insuficientes para exercer o número de atividades, envio de códigos incorretos, rasuras nos documentos ou preenchimento ilegível, preenchimento incompleto ou outras falhas no processo. ${ }^{(14)}$

Mensalmente todos os auditores elaboram um relatório descrevendo e contabilizando todas as atividades acompanhadas ao longo do mês, tanto numericamente, quanto qualitativamente. Nesse contexto, o enfermeiro auditor exerce papel notório, pois guia, coordena e lidera a equipe no processo de auditoria, demonstrando a necessidade deste profissional estar em constante atualização de conhecimentos para contribuir substancialmente para a melhora da qualidade da assistência ainda que indiretamente..$^{(13)}$

Outra função destinada aos enfermeiros auditores é participar ativamente na elaboração e avaliação dos contratos, tanto dando respaldo técnico com informações coletadas in loco e nos relatórios, quanto participar das avaliações junto com a diretoria da DRAS e a diretoria dos serviços contratualizados.

Realizaram-se também adequações no processo de trabalho da equipe, como o rodízio de auditores nas instituições contratualizadas, o que contribuiu para o desenvolvimento dos profissionais, por meio da troca de experiências, vivências, maior respaldo técnico para contribuir na elaboração de protocolos, documentos descritivos e contratos.

Nota-se que a auditoria se revela como uma ferramenta essencial para o funcionamento do SUS, na busca pela qualidade dos serviços prestados à população aliado ao emprego adequado da verba pública destinada à saúde. Além disso, a auditoria pode apoiar os gestores na identificação de necessidades eminentes, apuração de possiveis irregularidades que estejam prejudicando o serviço e estruturação de planos de ação para a correção ou adequação dos problemas levantados. ${ }^{(15)}$

Destaca-se que a operacionalização da auditoria na forma concorrente proporciona que o auditor esteja in loco na unidade de atendimento em que o paciente se encontra e em contato com a equipe de Enfermagem e Médica, visualizando divergências nas anotações, sanando dúvidas dos profissionais, observando a qualidade da assistência prestada, visitando o paciente quando necessário, auxiliando na otimização de alguns processos burocráticos que envolvem o SUS, dentre outros. ${ }^{(7)}$

Em relação a prática do processo de auditar infere-se que ele apresenta grande valia, influenciando diretamente nos custos e qualidade ao usuário da saúde. A auditoria como instrumento para a excelência da gestão em saúde não pretende em sua primazia apenas identificar anormalidades, mas sim ajustar. ${ }^{(12)}$

Atualmente na DRAS existem auditores de diversas categorias profissionais, como enfermeiros, médicos, fisioterapeutas e fonoaudiólogos, porém sendo apenas os primeiros com cargo instituido. A auditoria em saúde por meio do trabalho de equipes multiprofissionais pode contribuir para a melhoria na qualidade da assistência prestada, podendo gerar indicadores de eficiência e efetividade do serviço de saúde e atuar na otimização dos recursos financeiros, em que cada profissão pode desvelar as necessidades do seu campo de atuação, promovendo a integralidade da assistência multiprofissional, adequada e de qualidade, ${ }^{(16,17)}$ servindo de embasamento para a instituição de cargos de auditores em outras profissões, para além da enfermagem

\section{Limitações do estudo}

Considera-se como limitação desse estudo o remoto acesso a totalidade de documentos que compuseram o processo de implantação da auditoria no município, pois trata-se de um processo amplo que lança mão de muitos documentos.

Outra limitação é que o método utilizado impossibilita generalizar os resultados obtidos, sendo a realizada investigada para o municipio em questão.

\section{Contribuições do estudo para a prática}

Este trabalho pode servir como fonte de consulta, apoio e embasamento para futuros processos de implantação da auditoria operativa em outros municípios. Permite verificar o papel do enfermeiro no processo de implantação da auditoria operativa e de que forma está inserido nessa atividade. Além disso, este trabalho estimula o desenvolvimento de outras pesquisas sobre o tema, divulgando os resultados alcançados com a implantação do serviço.

\section{CONCLUSÃO}

Após a implantação e implementação da auditoria operativa em Enfermagem no SUS na Autarquia Municipal de Saúde do município em estudo, foi possivel observar por meio dos documentos analisados que esse serviço apresentou um papel fundamental na solidificação da qualidade dos serviços prestados nas instituições: A realização deste processo culminou no alcance de indicadores de qualidade, anteriormente não monitorados, com benefícios diretos à população assistida nos serviços auditados. Melhor cumprimento dos seus princípios e diretrizes, acompanhando o desenvolvimento das ações e serviços dirigidos à população, primando pela qualidade da assistência prestada. 
Diariamente são monitoradas, colhidas e analisadas uma grande quantidade de informações vitais no que tange a assistência à saúde dos usuários SUS. Esses dados têm subsidiado discussões entre os gestores do município para o planejamento e melhorias nas políticas e regulamentações na área da atenção hospitalar da região e, consequentemente, indicando possiveis fragilidades de outras áreas da saúde, como na atenção primária.

Ressalta-se que a implantação do serviço de auditoria operativa de enfermagem é de suma importância na gestão do SUS, não só na média e na alta complexidade, mas também na atenção primária e demais setores e serviços de assistência à saúde.
Participação dos autores: MCLH participou da concepção, análise, interpretação dos dados e redação do manuscrito. Responsável por todos os aspectos do trabalho na garantia da exatidão e integridade de qualquer parte da obra; VML participou da concepção do estudo, coleta, interpretação e análise dos dados, redação das versões do manuscrito, revisão crítica do conteúdo e aprovação da versão final; DRCP, RG, PSCP, ICKOC contribuiram com a revisão crítica relevante do conteúdo intelectual e aprovação final da versão a ser publicada.

\section{Conflito de Interesse: Não há}

\section{REFERÊNCIAS}

Barbosa DVS, Barbosa NB, Najberg E. Regulação em saúde: desafios à governança do SUS. Cad Saúde Colet [Internet] 2016 [cited 2018 jan 15]:24(1):49-54. Available from: http://www.scielo.br/pdf/ cadsc/2016nahead/1414-462X-cadsc-1414-462X201600010106.pdf

Peruzzo HE, Liberatti VM, Rossaneis MA, Vannuchi MTO, Haddad MCL. Perspectivas, sentimentos e vivências de ouvidores do Sistema Único de Saúde no exercício da função. Cienc Cuid Saude [Internet] 2017 [cited 2018 mai 24]:16(1). Available from: http://www.periodicos.uem.br/ojs/index.php/ CiencCuidSaude/article/view/36614/19407

Viana CD, Bragas LZT, Lazzari DD, Garcia CTF, Moura GMS. Implantação da auditoria concorrente de enfermagem: um relato de experiência. Texto \& Contexto Enferm [Internet] 2016 [cited 2018 fev 25];25(1):e3250014. Available from: http://www.scielo.br/pdf/tce/v25nl/pt_0104-0707tce-25-01-3250014.pdf.

Silva KR, Lima MDO, Sousa MA. Auditoria: ferramenta de enfermagem para melhoria da qualidade assistencial. Rev Gest Saúde [Internet] 2016 [cited 2018 mar 15];07(02): 793-810. Available from: http://periodicos.unb.br/index. php/rgs/article/view/22055

Júnior NJO, Cardoso KE. O papel do enfermeiro frente à auditoria hospitalar. Rev. Adm. Saúde. [Internet] 2017 [cited 2019 out 07]; 17 (68). Available from: http://cqh.org.br/ojs-2.4.8/index.php/ras/article/view/52/65.

Dorne J, Volpato JH. Conhecimentos teóricos de auditoria em enfermagem. Rev Uningá Review [Internet] 2017 [cited 2017 dez 11];15(1):8. Available from: http://revista.uninga.br/index.php/uningareviews/article/ view/738

Instituto Brasileiro de Geografia e Estatística. Diretoria de Pesquisas DPE. Coordenação de População e Indicadores Sociais - COPIS. 2019. [cited 2019 jan 25]. Available from: http://cidades.ibge.gov.br/xtras/perfil. php?codmun $=411370$.

Secretaria de Estado da Saúde do Paraná. Plano Estadual de Saúde Paraná 2016-2019. Curitiba: SESA; 2016

Prefeitura de Londrina. Secretaria de Saúde de Londrina. Composição da Diretoria de Regulação da Atenção à Saúde. [cited 2019 jan 10] Available from: http://www.londrina.pr.gov.br/index.php?option=com_
content\&view=article\&id=5598Itemid=603

Jornal Oficial do Municipio de Londrina, Londrina, ano XV, n. 2169, 21 maio 2013 [Internet]. [cited 2018 mai 10]. Available from: http://www2. londrina.pr.gov.br/jornaloficial/images/stories/jornalOficial/jornal_2169_ assinado.pdf

Londrina. Edital de Concurso Público no 082/2013 - GPOS/DGTES/ AMS. [Internet] [cited 2018 abr 25]. Available from: http://www.londrina. pr.gov.br/dados/images/stories/Storage/sec_gestao/concursos/ edital_082_2013_ams/ams_edital082_concurso_1_.pdf

Oliveira EM, Silva FWF. Dilemas no processo de auditoria: paradoxos dos serviços em saúde. Revista da Universidade Vale do Rio Verde [Internet] 2019 [cited 2019 out 07];17(1). Available from: http://periodicos.unincor.br/ index.php/revistaunincor/article/view/4973.

Tiburcio APN, Sousa LAA, Santos RFdos. A Importância do Enfermeiro Auditor nas Instituições Hospitalares. Rev. Psicol Saúde e Debate [Internet] 2019 [cited 2019 out 07];5(1):50-59. Available from: http://psicodebate. dpgpsifpm.com.br/index.php/periodico/article/view/V5N1A5/268.

Andrade AFSMde, Benevides LNB. Custo efetividade da auditoria concorrente em uma operadora de saúde. Rev. Adm. Saúde [Internet] 2019 [cited 2019 out 07];19(75): el62. Available from: http://www.cqh.org.br/ojs2.4.8/index.php/ras/article/view/162/271

Santos EO, Eslabão AD, Práticas de Auditoria no Sistema Único de Saúde: Uma Revisão Integrativa. Rev Fund Care Online. [Internet] 2019 [cited 2019 out 07];11(3):792-800. Available from: http://dx.doi.org/10.9789/21755361.2019.vlli3.792-800.

Quevedo ALAde, Leal RM. Auditoria em saúde e economia da saúde: análise de um estudo de caso. Cad. Ibero-amer. Dir. Sanit. [Internet] 2019 [cited 2019 out 07]:8(2): 1-132. Available from: http://dx.doi.org/10.17566/ciads.v8i2.503.

Santos ATS, Oliveira Cbde, Passos MdoC, Andrade AdaSA, Gallotti FCM. Integralidade do Cuidado na Formação do Enfermeiro: Visões e Vivências do Acadêmico de Enfermagem. Enferm. Foco [Internet] 2019 [cited 2019 out 07];10(1): 122-126. Available from: http://revista.cofen.gov.br/index. php/enfermagem/article/view/1397/507.

RECEBIDO:08/10/2019 - ACEITO: 12/02/2020 